\title{
Use of VA ECMO and percutaneous palliation of ductal dependent coarctation in a neonate with trisomy 21 and COVID-19 pneumonia
}

\author{
Sandeep Sainathan ${ }^{1} \cdot$ Martha Elisabeth Heal $^{2} \cdot$ Elman Frantz $^{2} \cdot$ Pace Johnston $^{2} \cdot$ Rebecca Smith $^{3} \cdot$ Mahesh Sharma $^{1}$
}

Received: 5 March 2021 / Revised: 17 April 2021 / Accepted: 20 April 2021 / Published online: 26 May 2021

(C) Indian Association of Cardiovascular-Thoracic Surgeons 2021

\begin{abstract}
We present a case of a neonate with trisomy 21 , ductal-dependent aortic coarctation, and severe respiratory failure secondary to coronavirus disease 2019 (COVID-19) pneumonia. The neonate was managed with venoarterial extracorporeal membrane oxygenation (VA ECMO), palliative stenting of the coarctation, and a vascular plug occlusion of a large patent ductus arteriosus. The patient was successfully weaned off extracorporeal membrane oxygenation (ECMO). The patient is currently awaiting a definitive surgical repair in the near future.
\end{abstract}

Keywords COVID-19 disease $\cdot$ ECMO $\cdot$ Aortic coarctation $\cdot$ Stent $\cdot$ Vascular plug $\cdot$ Down syndrome

\section{Introduction}

The coronavirus disease 2019 (COVID-19) is an ongoing pandemic caused by the severe acute respiratory syndrome coronavirus 2 (SARS-CoV-2). While the disease has been widespread and severe in adults, neonates are rarely infected [1]. We present a case of a neonate with severe COVID-19 pneumonia and congenital heart disease that was supported with venoarterial extracorporeal membrane oxygenation (VA ECMO) and the cardiac defect was palliated by a catheter intervention.

\section{Case report}

A 10-day-old, 3-kg male term-neonate with trisomy 21 was admitted to the pediatric cardiac critical care unit with a dusky

Sandeep Sainathan

surgeonsandeep@gmail.com

1 Department of Surgery, Section of Pediatric Cardiothoracic Surgery, University of North Carolina at Chapel Hill, 3040 Burnett- Womack building, Suite 3808, Chapel Hill, NC 27599, USA

2 Section of Pediatric Cardiology, University of North Carolina at Chapel Hill, Chapel Hill, NC, USA

3 Section of Pediatric Cardiac Critical Care, University of North Carolina at Chapel Hill, Chapel Hill, NC, USA appearance and poor femoral pulses. This was concerning for a coarctation of the aorta. An echocardiogram confirmed a coarctation of the aorta with moderate hypoplasia of the distal transverse aortic arch $(0.34$ to $0.29 \mathrm{~cm}$ from the left common carotid artery to the left subclavian artery) (Fig. 1a), a $0.7-\mathrm{cm}$ ascending and descending thoracic aorta, a bicuspid aortic valve, a small patent ductus arteriosus (PDA) with a bidirectional shunt, a small 4-mm atrial septal defect (ASD), and a small 3-mm perimembranous ventricular septal defect (VSD) both with a left to right shunt, a normal-sized mitral valve with trivial insufficiency, and a normal-sized left ventricle with normal function. Prostaglandin-E2 was started with the return of good femoral pulses, a systolic blood pressure gradient difference of 15-20 mmHg between the upper and lower extremity, and now a large PDA suggesting a ductal-dependent nature of the coarctation. There was no saturation difference between the extremities. The patient had respiratory congestion with secretions and fever. Given the ongoing COVID-19 pandemic and as a part of the respiratory viral workup, a COVID-19 test was done using the SAR-CoV-2 polymerase chain reaction (PCR) assay (Abbott Alinity, Lake Forest, IL) by a nasopharyngeal swab. The test came back positive and contact tracing revealed positivity in the mother and several other members of the family. The patient was nursed with precautions for COVID-19 disease including the use of a negative pressure room. While his cardiac condition stabilized on prostaglandin therapy with good biventricular function and good lower extremity perfusion, the patient's respiratory status gradually worsened including the need for supplemental 


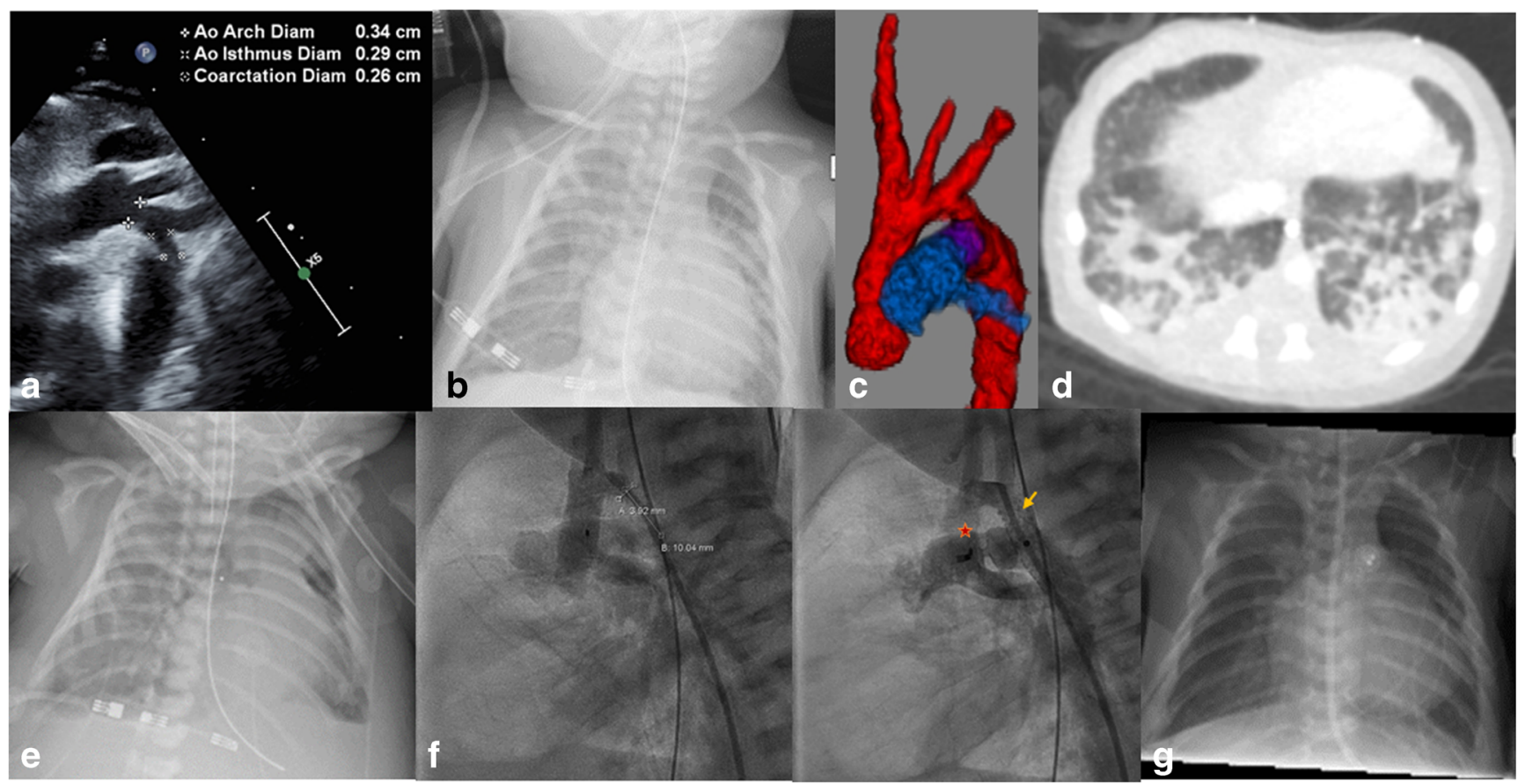

Fig. 1 a ECHO showing coarctation. b Chest X-ray showing infiltrates. c CT angiography showing coarctation, hypoplastic aortic arch, and PDA. d "Ground glass" infiltrates in CT chest. e Chest X-ray after VA ECMO cannulation. f Chest fluoroscopy (arrow, ductal stent; star, PDA plug). g Chest X-ray post ECMO decannulation with resolution of infiltrates and

oxygenation with the eventual need for intubation and mechanical ventilation. A chest X-ray showed infiltrates (Fig. 1b). A computed tomography (CT) scan of the chest, apart from nicely demonstrating the coarctation and hypoplastic aortic arch with a large PDA (Fig. 1c), showed ground-glass infiltration in the lung parenchyma concerning for COVID-19 pneumonia (Fig. 1d). The patient was given steroids for his COVID-19 disease. However, the patient had worsening of his respiratory failure with both severe hypoxia with preductal arterial saturation of $70 \%$ and $\mathrm{PaO}_{2}$ of $35-40 \mathrm{mmHg}$ and hypercarbia of $60-70 \mathrm{mmHg}$ with maximum conventional mechanical ventilator support. A trial on an oscillator was attempted with no improvement. He was also having acute renal injury with oliguria. Hence, the patient was cannulated for VA ECMO via his right cervical vessels (Fig. 1e). He stabilized on VA ECMO with flows up to $150 \mathrm{cc} / \mathrm{kg} / \mathrm{min}$. This resulted in a gradual resolution of the end-organ injury. Bivalirudin was used for anticoagulation. Given COVID-19 pneumonia, the patient was thought to be of a high surgical risk either for resection of the coarctation and arch augmentation with cardiopulmonary bypass, deep hypothermia and regional perfusion, or an alternative off-pump resection of the coarctation and extended end-to-end repair via a left thoracotomy. Hence, the patient was taken to the cardiac catheterization lab at $48 \mathrm{~h}$ of extracorporeal membrane oxygenation (ECMO) support after end organ recovery for stenting of his aortic coarctation with an Onyx (Medtronic, Minneapolis, vascular prostheses in situ. ECHO, echocardiogram; CT, computed tomography; PDA, patent ductus arteriosus; VA ECMO, venoarterial extracorporeal membrane oxygenation; ECMO, extracorporeal membrane oxygenation

$\mathrm{MN}$ ) drug-eluting $5 \times 15 \mathrm{~mm}$ coronary stent (Fig. 1f, arrow) and occlusion of his PDA with an 8-mm Amplatz vascular plug II (Abbott, Plymouth, MN) (Fig. 1f, star) via the femoral vessels. The procedure was successful with an $8 \mathrm{mmHg}$ residual mean gradient from the hypoplastic aortic arch, widely open stent, and a completely occluded PDA. The patient was eventually successfully weaned off of ECMO after 8 days of support with the repair of his right cervical vessels at the time of his decannulation and was extubated after 10 days from his time of decannulation. Bivalirudin was used for anticoagulation during the ECMO run and was continued after decannulation for the stents and was transitioned to aspirin once enteral feeding was initiated. A chest X-ray showed a gradual resolution of the infiltrates 4 weeks since the onset of symptoms (Fig. 1g). The future plan is to do a definitive surgical repair at about 4-6 months of age with resection of the coarctation, aortic arch augmentation, assess for the need for closure of the small perimembranous VSD, and closure of the ASD with cardiopulmonary bypass, deep hypothermia, and regional perfusion.

\section{Discussion}

The possible routes of transmissions of SARS-CoV-2 in the neonate include in utero, perinatal, and postnatal transmission and the data about this is still evolving [1]. In one 
multinational study, the perinatal transmission was estimated to be around 3\% and there is a suggestion that neonates with comorbidities and prematurity are more susceptible $[1,2]$. Also, COVID-19 disease may run a severe course in trisomy 21 [3]. The most likely mechanism is from respiratory droplet transmission from the infected mother to the neonate, and thus infection prevention measures such as wearing a mask and hand hygiene are recommended. Most neonatal infections are asymptomatic. However, severe illnesses such as acute respiratory distress syndrome (ARDS) and multi-organ dysfunction from viremia have been reported $[1,2]$. The treatment in the symptomatic neonate is supportive with unknown efficacy of drugs like remdesivir in the neonate. Also, it has emergency use authorization only for patients weighing at least $3.5 \mathrm{~kg}$. This neonate was in a unique situation. While the PDA was essential given the ductal-dependent nature of the coarctation, its presence was causing pulmonary overcirculation and thus likely further exacerbating the COVID-19 pneumonia. In addition, the PDA was causing renal injury secondary to visceral hypoperfusion by facilitating a systemic runoff into the pulmonary circulation. Also, the increased intrathoracic pressure from increasing mechanical ventilator support was causing systemic abdominal venous congestion and thus further exacerbating renal hypoperfusion by decreasing the renal perfusion pressure. To eliminate the PDA, the coarctation and hypoplastic aortic arch needed to be addressed first given the ductal-dependent nature of the coarctation. This could have been done either via a left thoracotomy, resection of the coarctation, and extended end-to-end repair with single lung ventilation or alternatively by a more complete approach using median sternotomy with cardiopulmonary bypass, deep hypothermia, and regional perfusion to repair the aortic arch and the coarctation with a patch. However, given the poor respiratory status and uncertain evolving clinical course of the COVID-19 disease, the patient was thought to be of a high surgical risk. Hence, we decided with stenting of the coarctation and vascular plug occlusion of the PDA as an alternative temporizing measure before a future definitive surgical repair. Before stenting, the patient had to be stabilized by breaking the vicious cycle of systemic arterial hypoperfusion from the PDA runoff and systemic venous hypertension from increasing mechanical ventilator support leading to end-organ injury such as an acute renal failure. ECMO was used to break this cycle by resting the lungs, decreasing the intrathoracic pressure, augmenting the cardiac output, and providing hemodynamic stability to safely perform the percutaneous procedure. Palliative stenting with coronary stents to treat critical aortic coarctation has been previously used in neonates with low birth weight (1500 g), prematurity, or experiencing multi-organ dysfunction as a palliation to a later definitive surgical correction [4-7]. The femoral artery was used for access (such as in our case) with the axillary artery being an alternative and 4- to 5-mm coronary stents were commonly used $[5,6]$. The mean gradients after stenting in these studies were about $5 \mathrm{mmHg}$. Overall neonatal palliative stenting is largely successful with most surviving and receiving definite surgical repair between ages of 3 and 4 months. The complexity of the future surgical repair does increase with the stenting because of the stent material in the aorta. However, with experience from arch augmentation after hybrid palliation for conditions such as hypoplastic left heart syndrome, we have developed reasonable surgical experience. The technique involves trimming as much of the stent material as possible and passing the suture between the interstices of the stent during the patch augmentation. Difficult areas may need to be buttressed additionally. Our case is unique with the use of ECMO and presence of COVID-19 disease as compared to the previously described patients [4-7]. The patient could have been managed with venovenous extracorporeal membrane oxygenation (VV ECMO) support for respiratory function. However, currently, there is no suitable cannula available to deploy VV ECMO in a neonate of this size. Besides, VA ECMO allowed for safe performance of the therapeutic catheterization procedure by providing hemodynamic stability, helped in the recovery of the lung function, and broke the previously described vicious cycle leading to endorgan injury. To note, it is important to flow high on VA ECMO (in the range of $150 \mathrm{cc} / \mathrm{kg} / \mathrm{min}$ ) with a large PDA because of the pulmonary steal. Careful monitoring of blood pressure at the upper and lower extremity is needed to balance arch hypertension proximal to the coarctation segment against lower body hypoperfusion distal to it.

\section{Conclusion}

This case report represents the utility of VA ECMO to promote lung recovery and provides hemodynamic stability in a neonate with ductal-dependent coarctation of the aorta and concomitant respiratory failure secondary to COVID-19 pneumonia. The relative stability achieved with VA ECMO allowed for the safe and effective performance of a percutaneous intervention prior to a definitive surgical intervention.

\section{Funding None.}

\section{Declarations}

Ethics approval Case reports are IRB exempt.

Informed consent We have written informed consent from the parent in compliance with Healthcare Insurance Portability and Accountability ACT of (HIPAA) 1996 regulation. We confirm that no portions of the manuscript and the submitted pictures have any information that can lead to identification of the patient. 
Statement of human and animal rights This is a retrospective chart review and is IRB exempt. The study was not part of any research protocol. The Onxy coronary stents are FDA approved for human implantation and are considered "off-label" use in pediatrics. Informed consent was obtained for their implantation.

Conflict of interest The authors declare no competing interests.

\section{References}

1. Barrero-Castillero A, Beam KS, Bernardini LB, et al. COVID-19: neonatal-perinatal perspectives. J Perinatol. 2020. https://doi.org/10. 1038/s41372-020-00874-x.

2. Shalish W, Lakshminrusimha S, Manzoni P, Keszler M, Sant'Anna GM. COVID-19 and neonatal respiratory care: current evidence and practical approach. Am J Perinatol. 2020;37:780-91.

3. Emami A, Javanmardi F, Akbari A, Asadi-Pooya AA. COVID-19 in patients with down syndrome. Neurol Sci. 2021:42:1649-1652.
4. Stegeman R, Breur JMPJ, Heuser J, et al. Primary coronary stent implantation is a feasible bridging therapy to surgery in very low birth weight infants with critical aortic coarctation. Int J Cardiol. 2018;261:62-65.

5. Haponiuk I, Chojnicki M, Steffens M, et al. Miniinvasive interventional bridge to major surgical repair of critical aortic coarctation in a newborn with severe multiorgan failure. Wideochir Inne Tech Maloinwazyjne. 2013;8:244-8.

6. Bugeja J, Cutajar D, Zahra C, Parascandalo R, Grech V, DeGiovanni JV. Aortic stenting for neonatal coarctation of the aorta - when should this be considered? Images Paediatr Cardiol. 2016;18:1-4.

7. Sreeram I, Sreeram N, Bennink G. Palliative stent implantation for coarctation in neonates and young infants. Ann Pediatr Cardiol. 2012;5:145-50.

Publisher's note Springer Nature remains neutral with regard to jurisdictional claims in published maps and institutional affiliations. 